\title{
Evolutionary Histories of Dwarf Galaxies in the Local Group
}

\author{
Eva K. Grebel \\ UCO/Lick Obs., University of California, Santa Cruz, CA 95064, USA
}

\begin{abstract}
The star formation histories of Local Group (LG) dwarf galaxies and more distant potential LG members are reviewed. Problems in defining the spatial extent of the LG and membership are briefly discussed. The morphological types found in the LG are presented, and it is suggested that we see continuous evolution from low-mass dwarf irregulars (dIrrs) to dwarf spheroidal galaxies (dSphs) in the LG.

Star formation histories for LG dwarfs and nearby LG candidates are compiled using population boxes. No two dwarfs, irrespective of morphological type, show the same evolutionary history, and all vary widely in ages of their subpopulations and in their enrichment history. The lack of gas in dSphs and certain dwarf ellipticals (dEs) is puzzling, both with respect to their star formation histories and the expected mass loss from red giants, but a new photoionization scenario may reconcile these contradictions. Old populations, often spatially very extended, may be a common property of dwarf galaxies, though their fractions can be very small. Almost all types of dwarf galaxies studied in detail so far show spatial variations in ages and abundances such as radial age/metallicity gradients. The observed star formation histories impose constraints on merger and accretion scenarios.

Properties of the Milky Way dwarf spheroidals are compared to the M31 dSphs and discussed in the framework of the ram pressure/tidal stripping scenario. It is demonstrated that the newly discovered LG dwarfs follow the same relationship for central surface brightness, mean metallicity, and absolute magnitude as the other LG dwarfs.
\end{abstract}

\section{Introduction}

Dwarf galaxies are the most frequent type of galaxy in the universe and a major constituent of galaxy groups and clusters. They play an important role in the formation and build-up of more massive galaxies through mergers and accretion. The surviving dwarf galaxies provide a fossil record of the early formation and evolutionary conditions of their parent clusters. Galaxies outside of clusters and groups record the evolution in isolated environments with a minimum of interactions. Accurate knowledge of their star formation and enrichment histories is paramount for understanding the evolution of clusters and void galaxies on cosmological time scales. Combining evolutionary histories with positional and 
orbital information helps to understand the impact of environment and mass on galaxy evolution.

Detailed studies of dwarf galaxy properties are only possible in sufficiently nearby, resolved galaxies - the dwarf galaxies of the Local Group (LG), whose properties and evolutionary histories will be summarized in this review. Other recent reviews of the star formation history of LG dwarfs include Grebel (1997), Da Costa (1998), Mateo (1998), and van den Bergh (1999).

For the purpose of this review, old refers to ages $>10 \mathrm{Gyr}$, intermediateage populations range from 1 to $10 \mathrm{Gyr}$, and young denotes populations younger than 1 Gyr.

\section{Galaxy Content of the Local Group}

We will consider all galaxies with $M_{B}>-18$ to be dwarf galaxies, i.e., all LG galaxies other than M31, the Milky Way, M33, and the LMC. However, the known dwarf galaxy census of the LG is incomplete and its spatial extent poorly defined. For a comprehensive picture of LG evolution it is important to understand these limitations.

\subsection{Morphological types and evolutionary transitions}

We distinguish four basic morphological galaxy types in the LG: spirals (S), dwarf irregulars (dIrr), dwarf ellipticals (dE), and dwarf spheroidals (dSph).

- The dIrrs are gas-rich, irregularly shaped galaxies with recent or ongoing star formation. Spiral density waves are absent.

- The dEs are compact, show very pronounced, dense, bulge-like cores, and may contain gas. They contain mainly old and intermediate-age populations and show in part recent star formation. Their surface brightness is typically $\mu_{V, 0}<21 \mathrm{mag} \operatorname{arcsec}^{-2}$, and $-18>M_{B}>-14$.

- The dSphs are the least luminous, least massive galaxies known and, surprisingly, are almost devoid of gas. They do not have a pronounced nucleus, show little central concentration and are dominated by old or intermediateage populations. In contrast to globular clusters dwarf spheroidals appear to be dark-matter dominated (Faber \& Lin 1983, Lin \& Faber 1983) with $6 \lesssim M / L_{V} \lesssim 100 M_{\odot}$ (Mateo et al. 1998). They are not rotationally supported. Typically $M \approx 2 \cdot 10^{7} M_{\odot}$ (Mateo et al. 1998), $\mu_{V, 0}>22 \mathrm{mag}$ $\operatorname{arcsec}^{-2}$ and $M_{B}>-14$ (Gallagher \& Wyse 1994).

But are the distinctions between the different morphological types really so well defined? dEs and dSphs follow the same scaling relations (e.g., Ferguson $\&$ Binggeli 1994) and are often considered the same type of galaxy. Low-mass dIrrs may eventually turn into dSphs. Transition types (dIrr/dSph) appear to show this evolution in progress. Like dSphs, these galaxies are dominated by old populations but contain gas and show some recent star formation. All these intermediate types are at distances $>250 \mathrm{kpc}$ from the large spirals and are probably less influenced by ram pressure stripping or tidal stripping. 


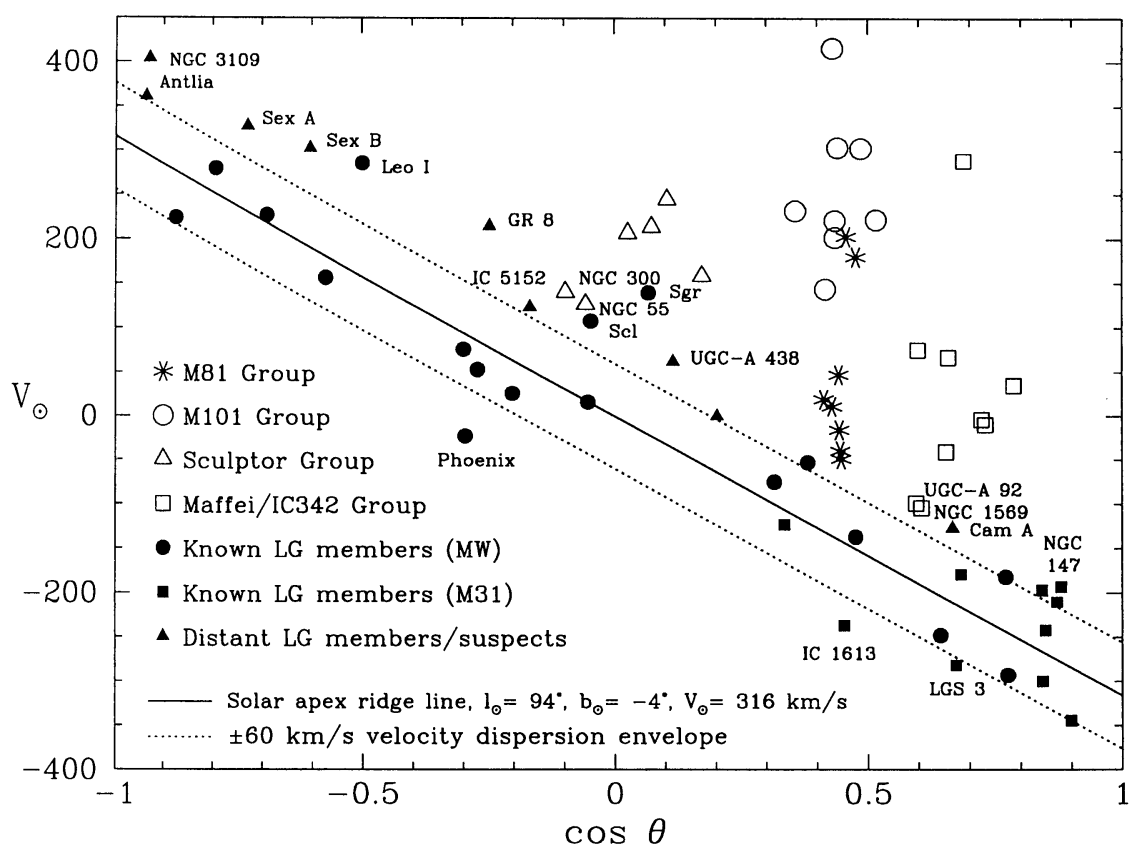

Figure 1. Heliocentric velocity, $V_{\odot}$, vs. cosine of the angle $\theta$ between solar apex and $l, b$ of a galaxy. The solid line represents the adopted solar motion from Karachentsev \& Makarov (1996). The dashed lines indicate the $\pm 60 \mathrm{~km} \mathrm{~s}^{-1}$ velocity dispersion of the Local Group (Sandage 1986). Distant Local Group suspects and nearby groups have positive velocity residuals due to cosmological expansion. Nearby Local Group dwarfs with large radial velocities also show positive residuals with respect to the $\pm 60 \mathrm{~km} \mathrm{~s}^{-1}$ velocity envelope.

The recent detection of gas around the Sculptor dSph galaxy (Carignan et al. 1998), the unexpected discovery of a young $(\sim 100-200 \mathrm{Myr})$ population in the Fornax dSph (Stetson et al. 1998), and of a $\sim 400-500$ Myr population in Leo I (Gallart et al. 1999) changes the traditional picture of dSphs as gasdevoid galaxies dominated by old or intermediate-age populations. Instead it seems that we see continuous evolution from low-mass dIrrs to dSphs, whose details may depend on the mass of the individual galaxy and its distance from the massive spirals. In contrast, blue compact dwarf (BCD) galaxies, which are rotationally supported systems, are unlikely to evolve into $\mathrm{dE} / \mathrm{dSph}$ galaxies unless they can somehow lose their angular momentum (van Zee et al. 1998).

In or near the LG additional galaxy types are found. The dIrr IC 10 may qualify as a starburst galaxy since its number of Wolf-Rayet stars is unusually high for a galaxy of its size; its WR star density is twice as high as in any other LG galaxy (Massey \& Armandroff 1995). The starburst activity may be triggered by gas accretion (Wilcots \& Miller 1998). The dIrr NGC 3109, a potential LG member depending on the adopted LG radius, may qualify as a low-surface-brightness spiral. Very extended spiral structure was detected by 
Demers et al. (1985), the surface-brightness is very low (Carignan 1985), and the galaxy's rotation curve is dominated by a dark component at nearly all radii (Jobin \& Carignan 1990). While the LG does not contain any BCD galaxies, the closest BCD, NGC 6789, is at a distance of only 2.1 Mpc (Drozdovsky \& Tikhonov 1999).

\subsection{Dynamical and distance considerations}

The location of galaxies within the $\pm 60 \mathrm{~km} \mathrm{~s}^{-1}$ velocity dispersion envelope in a $\cos \theta, V_{\odot}$ diagram (cosine of the angle between solar apex and $l, b$ of a galaxy versus its heliocentric velocity: Fig. 1) can be used as preliminary membership indicator (e.g., van den Bergh 1994, Grebel 1997). This method suggested LG membership for Schmidt's \& Boller's (1992) LG suspect AM 1001-270 (Grebel 1997), which was shown to be a possible LG member through a distance determination by Whiting et al. (1997), who named it Antlia after its parent constellation. For Leo A the diagram also predicted membership, and the revised distance to this dwarf galaxy (Tolstoy et al. 1998) is consistent with that. On the other hand, nearby dwarf galaxies with large radial velocities such as Leo I (Zaritsky et al. 1989; see Byrd et al. 1994 for an interesting interpretation), Sculptor, and the currently merging Sagittarius dwarf spheroidal galaxy (dSph) lie above the LG velocity dispersion boundary and would be regarded as non-members if distance information were lacking (Fig. 1). Galaxies with barycentric distances > 1.2 Mpc such as NGC 3109, Sex A, Sex B and GR 8, which are often considered LG members, also show positive velocity residuals with respect to the assumed LG locus. In contrast, the positions of IC 1613 and Phoenix indicate infall motion.

While distances give a better indication of LG membership it is not clear out to which distance galaxies may be considered members of the LG. Using the formalism of Yahil et al. (1977) and Sandage (1986), Grebel (1997) derived a radius of $1.8 \mathrm{Mpc}$ for a spherical, barycentric zero-velocity surface of the LG. However, the galaxies of the LG have an asymmetric, filamentary distribution (Karachentsev 1996), and the gravitational potential of the LG is likely to be nonspherical (Whiting, priv. comm.).

The simplified assumption of a sphere with $1.8 \mathrm{Mpc}$ radius implies overlap with other nearby groups such as the Sculptor group and the Maffei/IC 342 group. For instance, at a distance of $\sim 1.65 \mathrm{Mpc}$ from the barycenter of the LG NGC 55, a galaxy usually associated with the Sculptor group, lies within the zero-velocity surface for the LG (Mateo 1998), while dynamical calculations support Sculptor group membership (Whiting 1999). It is likely that the orbits of more distant LG members are affected by tidal forces of nearby groups, while outlying galaxies in nearby groups are influenced by the LG. Dunn \& Laflamme (1993) suggest that at redshifts of $z \geq 1$ tidal forces on LG galaxies were dominated by galaxies outside the LG.

LG membership can only be established for certain when a galaxy's orbit is known. Orbital information based on proper motions, however, is available only for six nearby Milky Way companions so far. In this review, star formation histories of a number of dwarf galaxies out to distances of $1.8 \mathrm{Mpc}$ from the LG barycenter will be discussed, though galaxies bound to the LG may fill a smaller volume. 


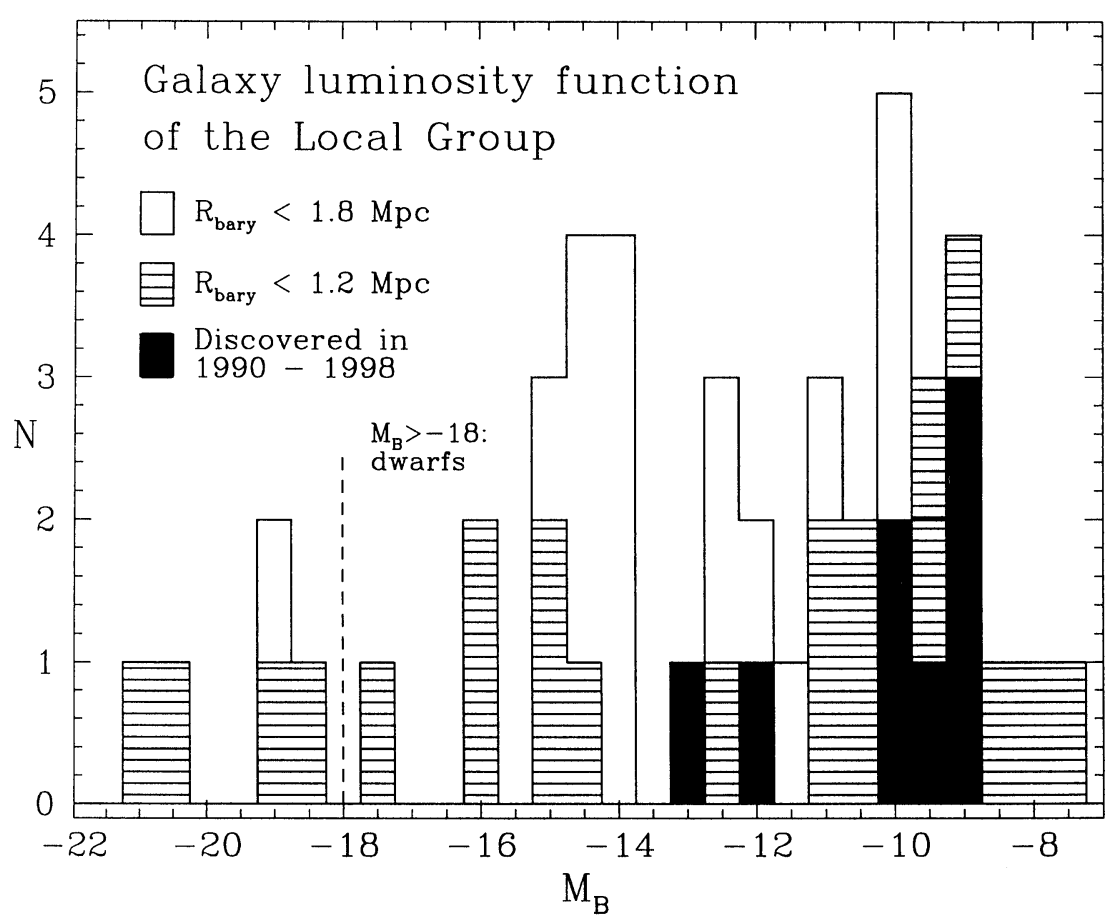

Figure 2. $B$-band luminosity function of galaxies within $1.8 \mathrm{Mpc}$ and 1.2 Mpc from the Local Group barycenter (excluding probable members of nearby groups). Data on the most recently detected Local Group dwarfs are from Karachentsev et al. (1999) and Grebel \& Guhathakurta (1999). $M_{B}$ for And V was estimated following Armandroff et al. (1998). Data for other galaxies was taken from Schmidt \& Boller (1992) and Mateo (1998). Note how the recent detections augment the faint end of the luminosity function, though it is still poorly populated in comparison to other nearby groups and clusters.

\subsection{Incompleteness of the dwarf galaxy census of the Local Group}

The LG is unique since here the low-mass end of the galaxy luminosity function can be studied in great detail. For example, dSph galaxies, which seem to be the dominant low-mass galaxy type, are undetectable at higher redshifts. A complete local dwarf census might allow us to determine the location and properties of the expected low-mass turnover or termination of the luminosity function.

The galaxy luminosity functions of nearby galaxy groups and clusters show a pronounced upturn for $M_{B}>-14 \mathrm{mag}$ (Trentham et al. 1998a), while the LG luminosity function (Fig. 2) is sparsely populated at its faint end. This may be in part an effect of small number statistics, but probably also reflects an incomplete local dwarf census. New faint dwarf galaxies are still being detected in the LG. In the past eight years alone eight new potential LG members were discovered (1990: Sextans, Tucana; 1994: Cam A, Sagittarius; 1997: Antlia; 1998: And V, 
And VI/Peg Dw, Cas Dw). Five of these are fainter than $M_{B}=-10$. Very low overall luminosity, low surface brightness, vicinity to a bright, superposed foreground star or a combination of these factors had prevented detection. Note that the only two known dwarf galaxies with $M_{B}>-8$ mag (Draco \& Ursa Minor) are nearby neighbors of the Milky Way. More still undetected galaxies of similarly low luminosity may lie at larger distances. The ongoing survey of Karachentseva and Karachentsev (1998) is revealing additional candidates, which we are following up on with the 2.4-m MDM telescope and the 10-m Keck telescopes (Guhathakurta et al. 1999). The galaxies studied so far are all at barycentric distances $>1.7 \mathrm{Mpc}$ and unlikely LG members, but they help to complete the dwarf census in the Local Volume. The Sloan Digital Sky Survey is expected to further increase the LG galaxy census. Velocity dispersion measurements of yet to be detected $M_{B}>-8$ galaxies that are sufficiently distant not to be tidally affected by the large spirals would show whether dSphs are indeed dark-matter dominated and whether $M \approx 2 \cdot 10^{7} M_{\odot}$ is the lower mass limit for dSphs.

Does the extreme low-mass end of the luminosity function consist of mere $\mathrm{H}$ I clouds that have not even formed stars yet? Some of the isolated, compact highvelocity clouds of Braun \& Burton (1999), with masses up to several $10^{7} M_{\odot}$ and infall motion toward the LG, might be progenitors of dwarf galaxies in accordance with the scenario of Blitz et al. (1999). They may also cause, or contribute to, star formation episodes through accretion, as appears to be the case for IC 10 (see Wilcots \& Miller 1998).

\section{Spatial Distribution of Local Group Dwarf Galaxies}

\subsection{Morphological segregation}

The spatial distribution of LG members and LG suspects shows morphological segregation (Fig. 3). An extended, scattered distribution of dwarf irregular galaxies (dIrrs) is also observed in other groups (e.g., Côté et al. 1997). The Tucana dSph is currently the only seemingly isolated dSph known in the LG, while all other dSphs are found within $<300 \mathrm{Mpc}$ of the large spirals. If this reflects a detection bias, then deep surveys should uncover additional dSphs at large distances. On the other hand, a deep survey of the Coma cluster (Trentham 1998b) shows a concentration and possibly confinement of the faintest dwarfs toward the cluster core. While the steep slope of the faint part of luminosity functions in richer clusters agrees with expectations from hierarchical cluster formation (Philipps et al. 1998), a different scenario has been proposed for the origin of Milky Way dwarf satellites.

\subsection{Polar orbital planes?}

The Milky Way's dwarf satellites and a number of halo globular clusters appear to lie along two (e.g., Majewski 1994) or more (Lynden-Bell \& Lynden-Bell 1995, Fusi Pecci et al. 1995) polar great circles, possibly indicating a common origin as remnants of at least two larger parent galaxies since accreted by the Milky Way. The available proper motions for LMC, SMC (Kroupa \& Bastian 1997), Draco, and Ursa Minor (Scholz \& Irwin 1994) appear consistent with motion along the 


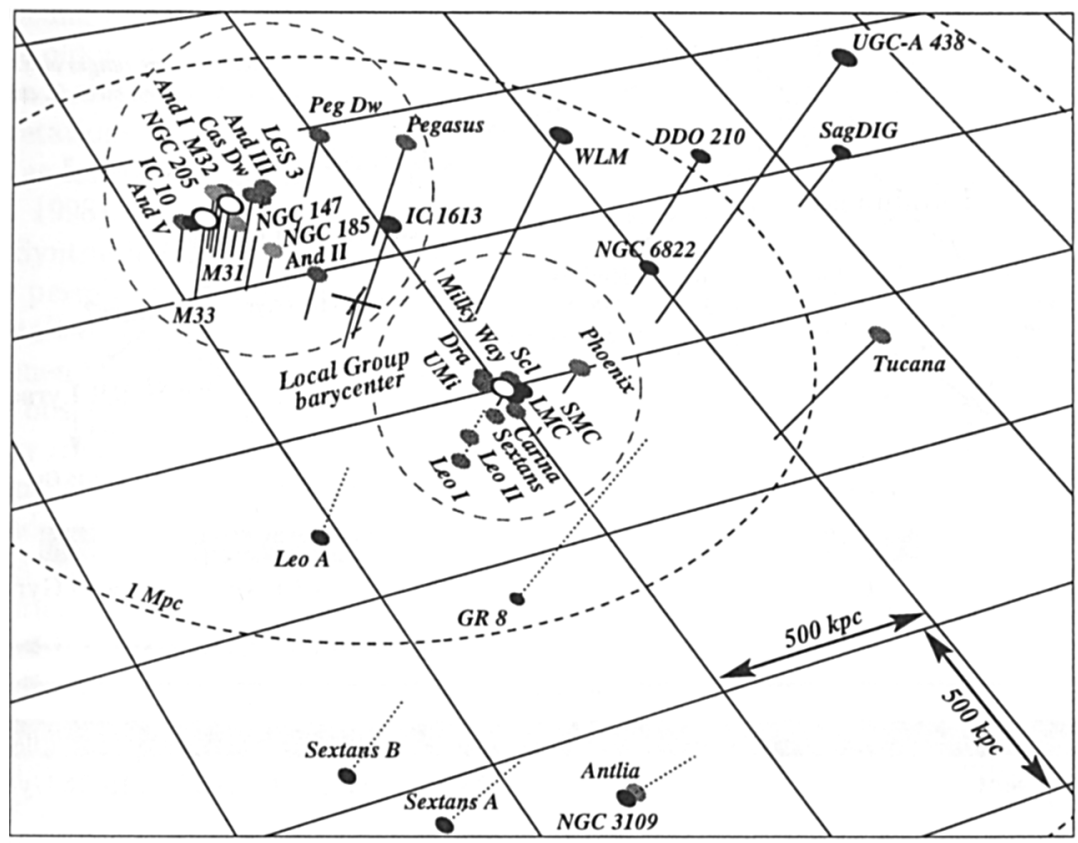

Figure 3. A scaled 3-D representation of the Local Group (LG). The dashed ellipsoid marks a radius of $1 \mathrm{Mpc}$ around the LG barycenter (cross). The underlying grid is parallel to the plane of the Milky Way. Galaxies above the plane are indicated by solid lines and below with dotted lines. The dashed circles enclose the presumed M31/M33 and the Milky Way subsystems. Morphological segregation is evident: the dEs and gas-deficient dSphs (light symbols) are closely concentrated around the large spirals (open symbols). DSph/dIrr transition types (e.g., Pegasus, LGS 3, Phoenix) tend to be somewhat more distant. Most dIrrs (dark symbols) are isolated and located at larger distances.

Magellanic stream, while the proper motion of Sculptor (Schweitzer et al. 1995) neither confirms or rules out motion along the proposed Fornax-Leo-Sculptor stream. Proper motions for the other more distant dSphs are not yet available.

Five out of six of M31's dSph companions as well as the dIrr/dSph LGS 3 and dIrr IC 10 appear to lie within $\pm 15^{\circ}$ of a potential Andromeda polar plane when transformed to a native $l_{M 31}, b_{M 31}$ coordinate system (Grebel et al. 1999a). For the M31 dSphs there are at present not even radial velocities available, and the existence of a polar orbital plane is purely speculative at this point.

\subsection{Constraints on the merger history}

In the merger remnant scenario one would expect that the epoch at which the disruption occurred should be marked by similar abundances in the galaxies associated with each polar plane. The Magellanic Clouds, Draco, and Ursa Minor can be tidal fragments of the same parent only if the break-up happened at a very early time; else the observed star formation and enrichment histories make 


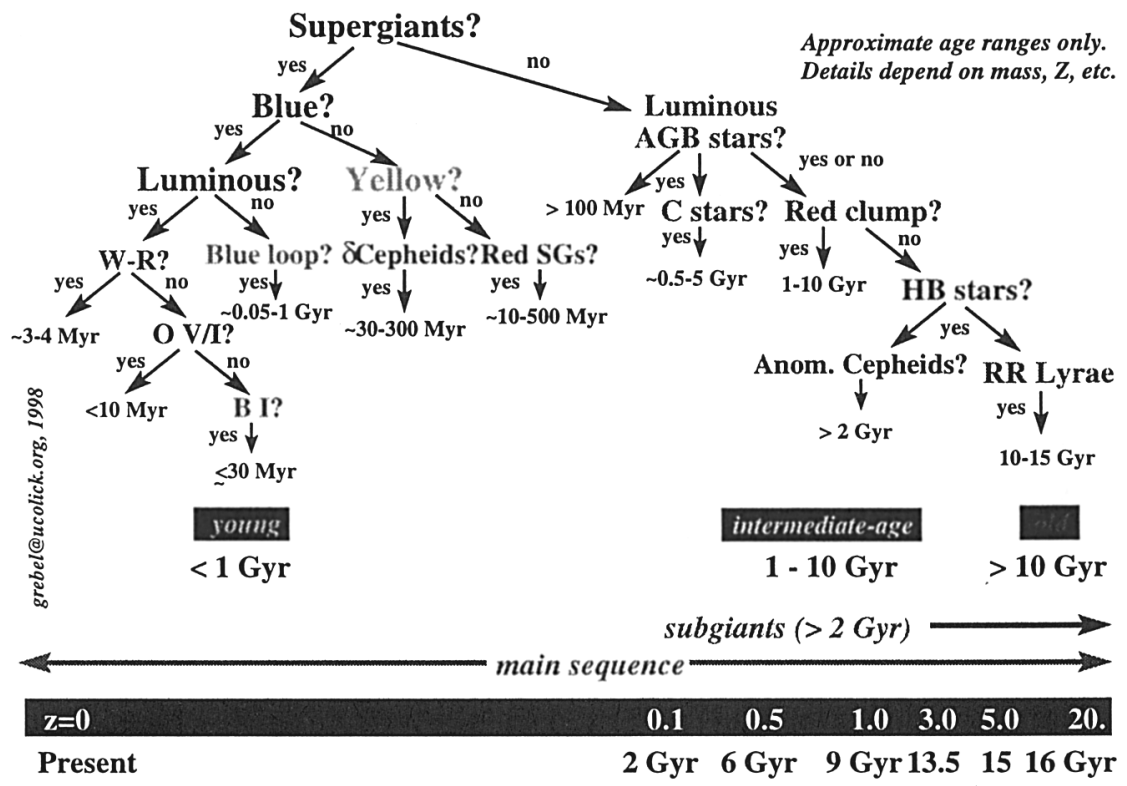

Figure 4. Stars as age tracers. Listed age ranges are rough estimates only. Note how the resolution decreases drastically with increasing age. The redshift-age axis was derived for $\mathrm{H}_{0}=50 \mathrm{~km} \mathrm{~s}^{-1} \mathrm{Mpc}^{-1}, \Omega=0.2$.

a common origin unlikely (Olszewski 1998). Unavane et al. (1996) concluded from a study of ages and abundances in Galactic halo field stars that the major merger epoch must have been $\gtrsim 10$ Gyr ago.

Only two of the Milky Way dSphs, Fornax and Sagittarius, contain globular clusters. They can contribute both globular clusters of the same age as the oldest halo globulars (Buonanno et al. 1998, Layden \& Sarajedini 1997), and clusters up to 4 Gyr (Fornax GC\#4, Marconi et al. 1999) or 7 Gyr younger (Sagittarius's Terzan 7, Chaboyer at al. 1996). The two most metal-poor Fornax globular clusters, GC\#5 (-1.9 dex) and GC\#1 (-2.0 dex), show the second-parameter effect (Smith et al. 1996), but neither Fornax's nor Sagittarius's globulars resemble the "young" halo clusters (Smith et al. 1998).

\section{Deriving Star Formation Histories}

Studies of the evolutionary history of a galaxy usually combine deep CCD photometry to derive color-magnitude diagrams (CMDs), and the use of stars as age tracers (Fig. 4). Where available this is supplemented by spectroscopic abundance information, ISM and kinematic information.

The determination of the star formation history of a galaxy is a multiparameter problem. The interpretation depends crucially on the knowledge of reddening (both foreground and internal extinction) and distance. Data may be compromised by crowding and high or variable internal extinction. With 
increasing distance it becomes more and more difficult to study the properties of the older populations. Mixed populations and the age-metallicity-reddeningdistance degeneracy present major challenges and may lead to ambiguous interpretations. The ambiguity can be reduced by using independent estimators such as foreground extinction from the IRAS/DIRBE reddening maps (Schlegel et al. 1998), or by using independent spectroscopic abundances.

Synthetic CMDs, sophisticated modelling techniques and statistical evaluations permit the reduction of the ambiguities to some extent and the extraction of detailed star formation histories (see Aparicio's 1999 review). It is important to remember that these analyses depend on how well evolutionary models reproduce observational parameters (Fig. 5) as well as on assumptions about IMFs, binary fractions, and metallicity evolution. Results obtained with different evolutionary models may not be directly comparable and tend to differ in ages, age spreads, and enrichment.

A way of testing these methods is through the creation of an artificial, yet realistic, stellar population composed of input data with well-determined (differential) ages and spectroscopic abundances, such as Galactic and Magellanic Cloud clusters corrected for distance and reddening. It would be interesting to see what star formation histories will be extracted through synthetic CMD methods by different groups. Several groups have expressed their willingness to participate in such a test, and we hope to carry out this experiment in the near future.

Special types of stars can be crucial for uncovering subpopulations even when high-quality CMDs are available. For instance, the presence of a small intermediate-age population may not be obvious from the CMD of an old dSph, but the detection of carbon stars traces this population unambiguously. RR Lyrae stars or blue horizontal branch stars indicate an old population (though their lack does not necessarily imply the absence of an old population since second-parameter effects can also play a role).

While photometry is the primary method for stellar population studies, spectroscopy provides accurate abundances, wind properties, spectral types, radial velocities, or stellar velocity dispersions. The new $10 \mathrm{~m}$-class telescopes will make many more LG galaxies accessible for spectroscopic studies and help to uncover enrichment histories and chemical evolution as well as internal kinematics and velocity dispersions. The planned astrometric satellite missions will increase and improve astrometric information for LG galaxies and help to derive their kinematics and orbits in the LG. Ultimately this will enable us to constrain past interaction events.

Star formation histories must be supplemented by studies of the ISM in and around galaxies - gas content and distribution, current star formation rates, kinematics, abundances (e.g., Young \& Lo 1997ab, Wilcots \& Miller 1998, Sage et al. 1998). The ongoing HIPASS H I multibeam survey (Staveley-Smith 1997) will allow the mapping of the large-scale distribution of $\mathrm{H}$ I in the $\mathrm{LG}$ and beyond and to search for extended $\mathrm{H}$ I clouds associated with dwarf galaxies (Carignan 1999). Deep ongoing $\mathrm{H} \alpha$ surveys will help to establish whether photoionized gas (Section 7.3) is associated with $\mathrm{dSph}$ galaxies and whether high-velocity clouds permeate the Local Group (Maloney \& Bland-Hawthorn 1999). 


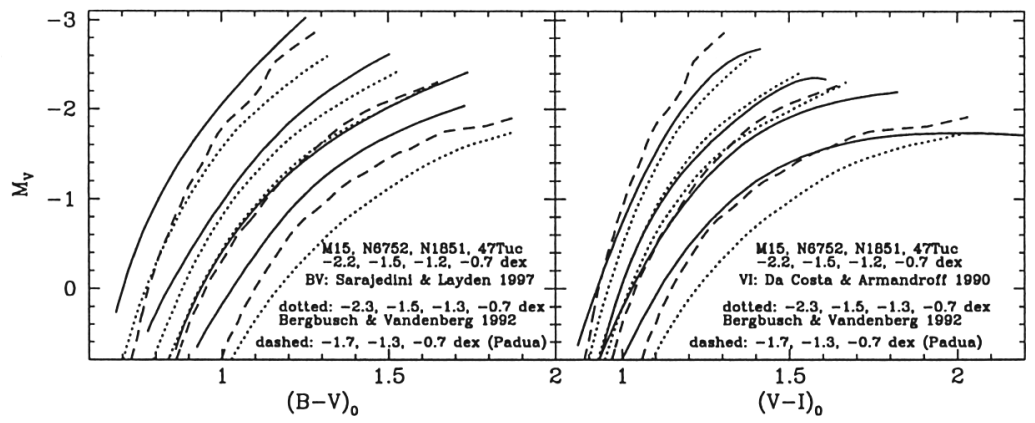

Figure 5. Comparison of the mean locations of observed red giant branches of Galactic globular clusters (solid lines: from Sarajedini \& Layden 1997 and Da Costa \& Armandroff 1990) with theoretical isochrones from Bergbusch \& VandenBerg (1992) and Bertelli et al. (1994). Note the good agreement for intermediate metallicities $([\mathrm{Fe} / \mathrm{H}]=-1.3 \mathrm{dex})$ and the discrepancies for higher and very low metallicities, which affect the results from synthetic color-magnitude diagram studies.

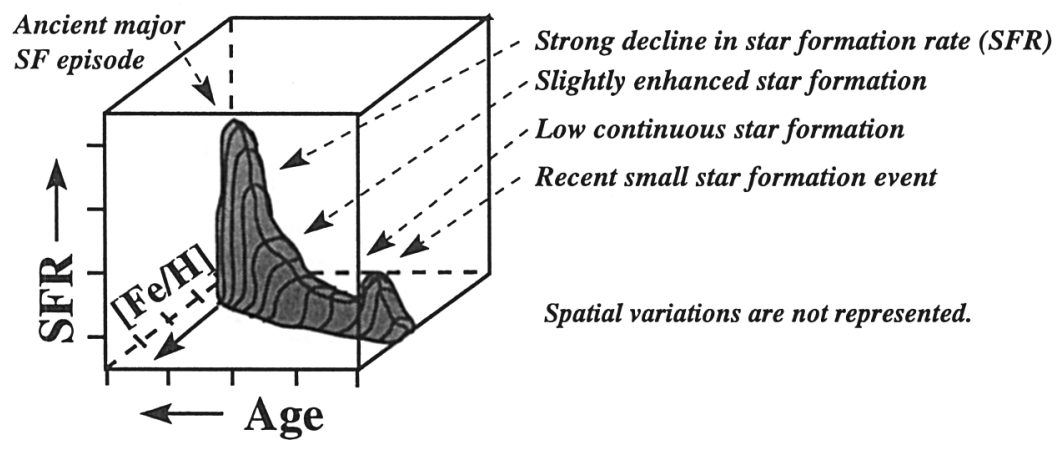

Figure 6. A sample population box for a fictitious galaxy.

\section{Star Formation Histories of Local Group Dwarfs}

\subsection{Population boxes}

Hodge (1989) introduced population boxes as a 3-D visualization of the evolutionary history of a galaxy (Fig. 6) as a function of time, star formation rate, and metallicity. Figures 7 and 8 show a compilation of star formation histories of LG galaxies based on data from sources using heterogeneous observational techniques and theoretical models (see Tab. $4 \& 5$ in Grebel 1997 for a compilation of properties)

Data on metallicities and enrichment are often uncertain. A galaxy may have a metallicity estimate for the old population from the slope of the RGB and an oxygen abundance determination from an $\mathrm{H}$ II region, which can be translated into a metallicity value (but see Richer \& McCall 1995 for caveats). In other cases abundance estimates are based solely on synthetic CMDs and 


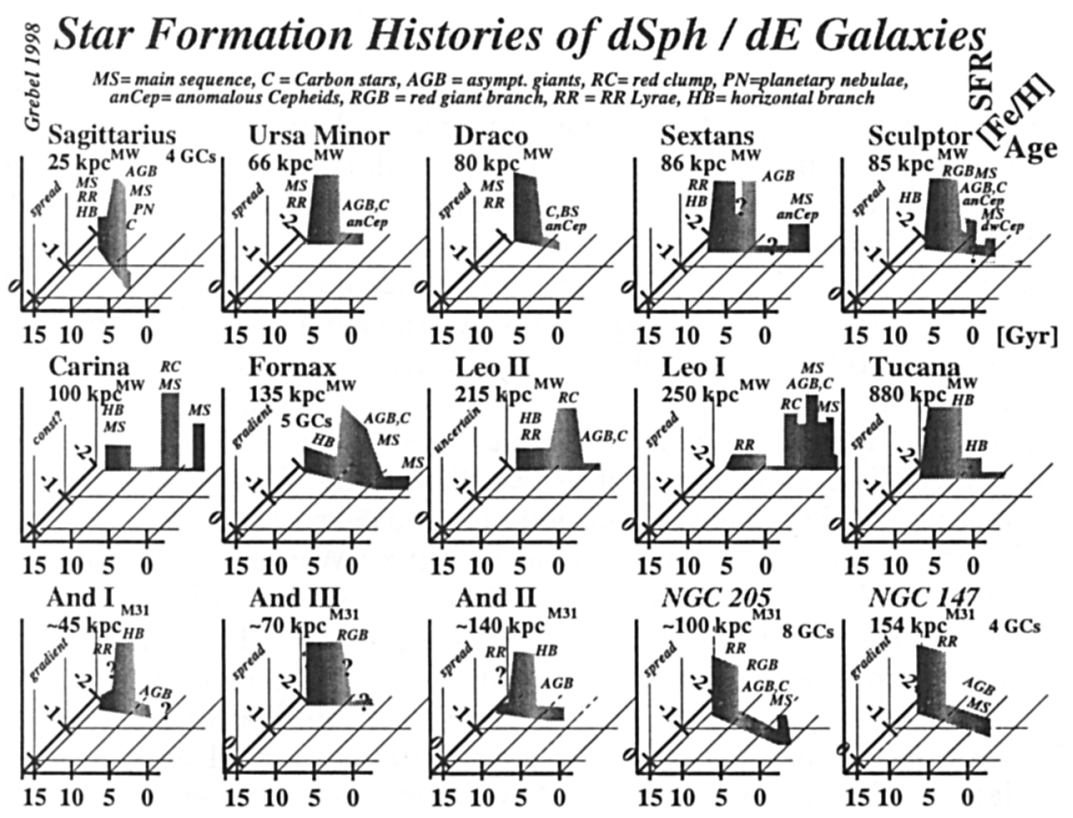

Figure 7. The upper two rows list population boxes for Milky Way dSphs (exception: Tucana) and their distances from the Milky Way. The bottom row distances refer to M31. For references, see Table 5 in Grebel (1997) and the following more recent studies: Marconi et al. 1998, Layden \& Sarajedini (1997), Grillmair et al. 1998, HurleyKeller et al. 1999, Hurley-Keller et al. 1998, Mighell 1997, Stetson et al. 1998, Stetson \& Grebel 1999, Gallart et al. 1999, Da Costa 1998, and Armandroff \& Da Costa 1999.

thus depend on the reliability of the input models (see Fig. 5). For only a few galaxies are there spectroscopically determined abundances for a large number of stars. Substantial metallicity spreads (e.g., $-3.0<[\mathrm{Fe} / \mathrm{H}]<-1.5$ dex in Draco, Shetrone et al. 1998) are found in the old populations of some dSphs, while other galaxies show very little evidence of metallicity spreads or enrichment. A galaxy that experienced hardly any enrichment despite repeated star formation episodes appears to be Carina (Smecker-Hane et al. 1994).

Time-dependent star formation rates (SFR) are not yet known for most galaxies. Therefore qualitative estimates of relative subpopulation ratios are used. It should be remembered that time resolution decreases with age and that we have very little information about details of star formation episodes longer ago than $\approx 10$ Gyr.

No two dwarf galaxies in the LG have the same star formation history. Some show pronounced enrichment, while others remain almost unchanged despite repeated episodes. Epochs and time scales of star formation episodes vary widely. 


\subsection{Ram pressure/tidal stripping}

For the 9 dSphs associated with the Milky Way (rows 1 and 2 in Fig. 7) the dominant populations tend to become younger with increasing distance from the Milky Way - a potential consequence of tidal/ram pressure stripping as suggested by Lin \& Faber (1983) and van den Bergh (1994). Interestingly, no such behavior is observed for the six known M31 dSph companions (Grebel \& Guhathakurta 1999), none of which appears to have a dominant intermediateage population even though though they span the same range of galactocentric distances as the Milky Way dSphs. Since we have no orbital information, we cannot exclude that the more distant dSphs are on eccentric orbits and were stripped of their gas in previous close passages. Also, M31 is more massive than the Milky Way, and more distant satellites may thus be affected by ram pressure stripping. The strong correlation between central surface brightness and present-day galactocentric distance found for Milky Way dSphs by Bellazzini et al. (1996) is much less pronounced for the M31 dSphs, and their central surface brightness values show a smaller range in magnitude. This may reflect the comparatively stronger tidal effects from M31 but is also caused by the lack of dominant intermediate-age populations. Note that the dIrr/dSph LGS 3 lies at a similar deprojected distance from M31 and contains a significant intermediateage population. The newly discovered M31 companions follow the relationship between central surface brightness, mean metallicity, and absolute magnitude as the other LG dSphs, dIrrs, and dEs (Fig. 9; Grebel \& Guhathakurta 1999).

\subsection{Old populations - ubiquitous?}

Old populations have been detected in most galaxies studied in detail so far. In galaxies dominated by intermediate-age populations (e.g., Leo A, Tolstoy et al. 1998) the presence of a small old population cannot be excluded. In dIrrs affected by crowding and internal extinction the usually more extended old populations have been detected through deep imaging of the halo regions (Minniti \& Zijlstra 1996, Minniti et al. 1999). It seems that there was a common epoch of the earliest star formation in at least some dwarf galaxies. Main-sequence photometry shows that the oldest globular clusters in the LMC, Sagittarius, and Fornax are as old as the oldest Galactic halo clusters (Olsen et al. 1998, Montegriffo et al. 1998, Buonanno et al. 1998). The same has been suggested for the oldest globulars in M31 and M33 (Sarajedini et al. 1998), while the SMC began to form globular clusters $\approx 3$ Gyr later (Mighell et al. 1998).

\subsection{Spatial variations in metallicity and star formation history}

Most LG dwarfs show position-dependent variations in metallicity and star formation history. For young populations in dIrr these variations are easily traced by the spotty distribution of $\mathrm{H}$ II regions. From an analysis of supergiants and Cepheids Grebel \& Brandner (1998) found the time scale for continuing star formation in the LMC within areas of $\lesssim 500 \mathrm{pc}$ to be of the order of $\lesssim 200$ Myr, similar to time scales observed in several dIrr galaxies (e.g., Dohm-Palmer et al. 1997: Sextans A; Dohm-Palmer et al. 1998: GR 8). In Sex A recent star formation progressed from the center outwards and currently takes place along the inside of an H I ring around the center (van Dyk et al. 1998). Similar central H I holes have also been detected in dIrr galaxies outside the LG (Puche \& 


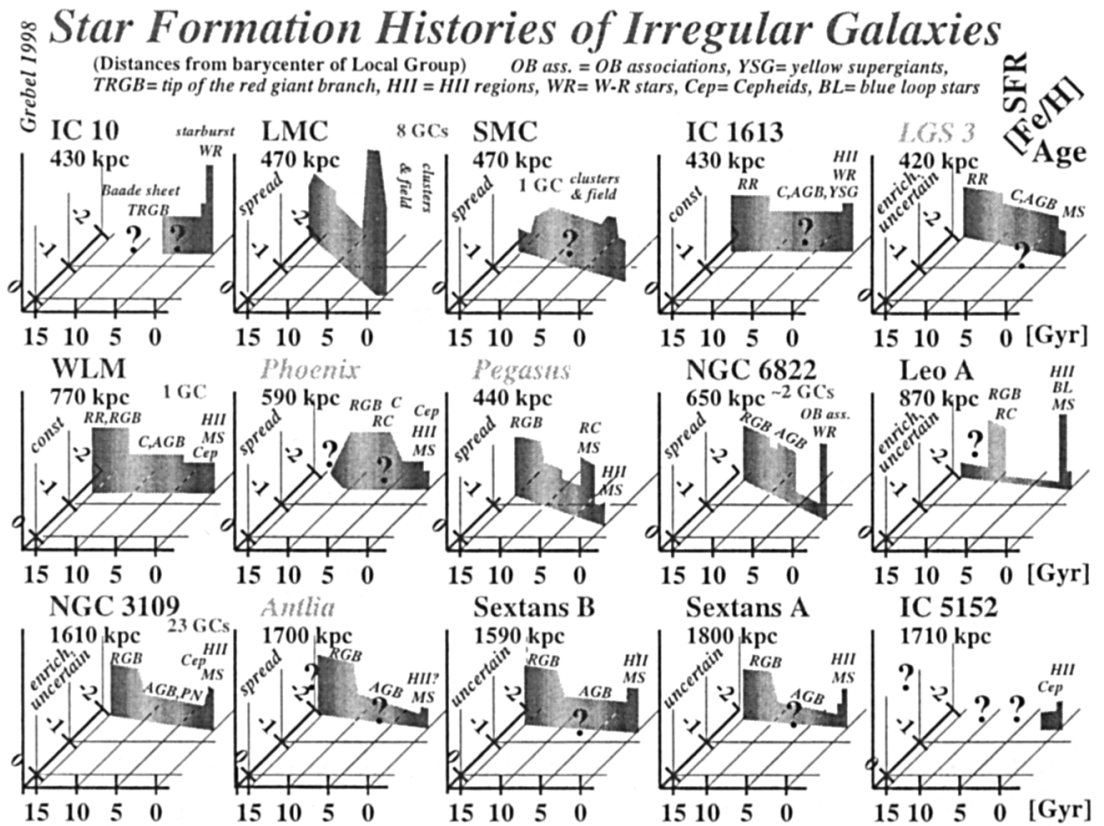

Figure 8. Star formation histories of dIrr and dIrr/dSph (italics) galaxies visualized through population boxes. For references, see Table 4 in Grebel (1997) and the following more recent studies: Geha et al. 1998, Grebel et al. 1999b, Aparicio et al. 1997b, Mould 1997, Minniti \& Zijlstra 1997, Martínez-Delgado et al. 1999, Gallagher et al. 1998, Aparicio et al. 1997c, Skillman et al. 1997, Tolstoy et al. 1998, Whiting et al. 1997, Aparicio et al. 1997a, Sarajedini et al. 1997, Dohm-Palmer et al. 1997, and van Dyk et al. 1998.

Westphal 1994). Depending on the shell propagation velocity, the density of the ambient medium, and the mass of the parent galaxies the gas may eventually be blown out.

Old populations in irregulars and dIrrs (e.g., Sex A: Hunter \& Plummer 1996, van Dyk et al. 1998; WLM: Minniti \& Zijlstra 1996; NGC 3109: Minniti et al. 1999) have been found to be much more extended than recent star formation events, which tend to be concentrated near the center of these galaxies. Extended old stellar "halos" have also been found around around transition types such as the dSph/dIrr Antlia (Aparicio et al. 1997a, Sarajedini et al. 1997), dSphs like And I (Da Costa et al. 1996), Carina (Mighell 1997), Fornax (Grebel 1997, Stetson et al. 1998, Grebel \& Stetson 1999) and Sculptor (Hurley-Keller et al. 1999), while younger populations are again confined to the central regions. 


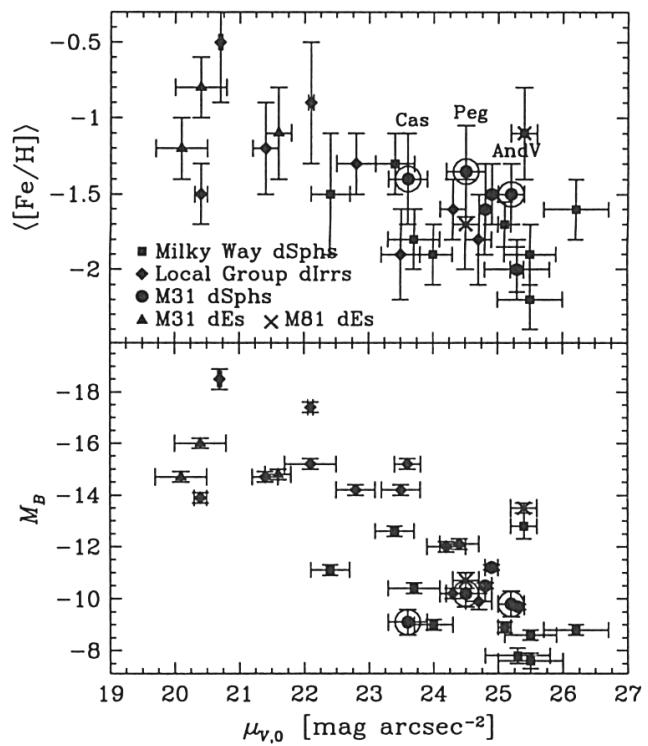

Figure 9. Mean metallicity, $\langle[\mathrm{Fe} / \mathrm{H}]\rangle$, plotted versus central surface brightness in the $V$ band, $\mu_{V, 0}$, for nearby dwarf galaxies (top), and the same for absolute $B$-band magnitude, $M_{B}$, versus $\mu_{V, 0}$ (bottom). The data are from Grebel (1997), Armandroff et al. (1998), Grebel \& Guhathakurta (1999), Mateo (1998), and Karachentsev \& Karachentseva (1998). The points representing the newly discovered M31 companions are circled. Note that they follow the mean $\langle[\mathrm{Fe} / \mathrm{H}]\rangle-\mathrm{vs}-\mu_{V, 0^{-}}$ vs- $M_{B}$ relation within the errors.

\section{Modes of Star Formation}

In low-mass galaxies the initial star formation episode may cause most of the gas to be expelled while more massive galaxies are able to retain their gas much longer or even continue to form stars over a Hubble time (Hunter 1997).

\subsection{Continuous star formation}

Slow, continuous star formation interrupted by short, inactive periods ("gasping star formation") was found in a number of the more massive LG dIrrs (NGC 3109: Greggio et al. 1993; Sex A: Aparicio et al. 1987; Sex B: Tosi et al. 1991; NGC 6822: Marconi et al. 1995, Gallart et al. 1996). Despite winds from massive stars and supernovae these galaxies are sufficiently massive to retain much of their gas and to undergo gradual enrichment (De Young \& Heckman 1994) through feedback from newly formed stars and gas recycling.

\subsection{Interactions, accretion, and triggered star formation}

Accretion plays an important role in providing star-forming material. Star formation with metal-poor accreted material is a possible explanation for the dis- 
tinct episodes observed in the Carina dSph (Smecker-Hane et al. 1994, HurleyKeller et al. 1998), the long pauses in between, and the lack of enrichment.

Young \& Lo (1997b) and Welch et al. (1998) found the gas in the dE NGC 205 to be rotating in contrast to the stars in this galaxy. Interaction with a gas cloud in the disk of M31 or accretion of an intergalactic H I cloud are likely explanations and may be responsible for the recent episode of star formation in NGC 205.

The dIrr IC 10 shows counter-rotating $\mathrm{H}$ I gas in its outer parts and is embedded in a very extended H I cloud (Wilcots \& Miller 1998), both likely signatures of recent and still ongoing accretion. The current starburst was probably triggered by this accretion event. Apart from extended tidal tails (e.g., Putman et al. 1998) and mutual disruption both Magellanic Clouds show enhanced cluster formation 100-200 Myr ago (Grebel et al. 1999b), coincident with the closest encounters between the Clouds, and with the Milky Way (Gardiner \& Noguchi 1996).

\section{Gas in/around Dwarf Ellipticals and Dwarf Spheroidals}

\subsection{Neutral gas}

NGC 185, a galaxy with ongoing star formation, is the only one of the four dE companion of M31 whose observed H I content is consistent with expectations from stellar mass loss (Sage et al. 1998). NGC 147 with predominantly old and intermediate-age populations (Han et al. 1997) contains less than $2 \%$ of the expected gas (Sage et al. 1998) and represents the most extreme case of gas deficiency among the dEs. Supernova explosions, interactions with, and passages through, the disk of M31 (Sofue 1994) may have stripped the M31 companions of part of their gas. As discussed by Sage et al. (1998), neither differences in mass nor evolutionary history can explain the observed differences in the gas content of the four M31 dEs. If NGC 147 and NGC 185 are a jointly formed, bound binary pair of comparable mass (van den Bergh 1998), the differing gas and dust content is even more puzzling.

The H I content of LG dSphs has upper limits of $10^{2}-10^{5} M_{\odot}$ (Knapp et al. 1978, Thuan \& Martin 1979, Mould et al. 1990, Koribalski et al. 1994). While a few supernova events may suffice to eject most of the gas of a low-mass dSph (Mac Low \& Ferrara 1998), these upper limits are even below the expected return from red giant mass loss. The lack of gas is particularly surprising in dSphs with repeated, well-separated episodes of intermediate-age star formation such as in Carina (Smecker-Hane et al. 1994, Hurley-Keller et al. 1998) or very recent star formation such as in Fornax (Stetson et al. 1998) and Leo I (Gallart et al. 1999).

Gas with $>3 \cdot 10^{4} M_{\odot}$ moving with the systemic velocity of Sculptor has been detected around this galaxy (Carignan et al. 1998; see also Knapp et al. 1978). The amount of gas is consistent with $10 \%$ of the estimated mass loss from RGB stars during the past 8-10 Gyr and may either have been ejected in an earlier star formation episode, stripped by Galactic tides (indeed the highest concentrations are found along Sculptor's direction of motion), or could be of external origin. The HIPASS survey is currently being used to search other dwarf galaxies for similar surrounding $\mathrm{H}$ I structures. Gas clouds rather than 
shells were detected near two other dwarf galaxies (Tucana: Oosterloo et al. 1996; Sagittarius: Bland-Hawthorn et al. 1998), but it is unclear whether these clouds are associated with the dwarfs.

\subsection{Heated/photoionized gas}

Type Ia supernovae may heat and disperse gas, which remains confined by the action of the dark matter halo of a dwarf galaxy (Burkert \& Ruiz-Lapuente 1997). After several Gyr the gas will have cooled down sufficiently to initiate a second epoch of star formation. The new generation should have higher metallicity after having been enriched by the Type Ia supernovae. While such time gaps are observed in Carina, the younger populations do not seem to have been enriched.

Lin \& Murray (1999) suggest a scenario in which newly formed massive stars and supernovae quench further star formation and ionize the residual gas, which expands into the outer regions of the dwarf galaxy. It is retained even if it is outside the tidal radius ( $\mathrm{Oh}$ et al. 1995), and continues to follow the orbit of the dwarf. The low density extends the cooling time beyond $1 \mathrm{Gyr}$ and the gas remains ionized through the extragalactic UV radiation field and radiation from the nearest spiral. The gas converges with the host galaxy at apogalacticon, where the exposure to the Galactic UV flux is minimized. After one or several apogalactic passages the gas may become sufficiently compressed to cool, recombine, and eventually form stars again. The Lin \& Murray scenario accounts for the non-detection of dSphs in $\mathrm{H}_{\mathrm{I}}$ and for long dormant periods without necessarily implying enrichment. Deep $\mathrm{H} \alpha$ Fabry-Pérot surveys are currently on their way to test the prediction of photoionized gas around dSphs.

\section{Summary}

The size of the LG and consequently its membership are poorly defined. The outer regions of nearby groups overlap with the outskirts of the LG. Knowledge of orbits is required to understand interactions with nearby groups and within the LG itself, and to reliably constrain membership.

The LG dwarf galaxy census is incomplete, and the faint end of the LG galaxy luminosity function is deficient in comparison to other nearby groups. Is the morphological segregation in the LG caused to some extent by a detection bias? Are there more distant, isolated dSphs like Tucana to be discovered?

In 1998 the discovery of three new dwarf galaxies was announced, all of which turned out to be distant dSph companion candidates of M31 (Armandroff et al. 1998, Karachentsev \& Karachentseva 1998, Tikhonov \& Karachentsev 1999, Grebel \& Guhathakurta 1999). The new dSphs follow the same relationship for central surface brightness, mean metallicity, and absolute magnitude as the other LG dwarfs. In contrast to Milky Way dSphs at comparable distances from the parent spiral, these dSphs do not show indications of dominant intermediate-age populations as one might have expected from the ram pressure/tidal stripping scenario (Lin \& Faber 1983, van den Bergh 1994), nor do they show the strong correlation between central surface brightness and galactocentric radius found for Milky Way dSphs (Bellazzini et al. 1996). Sensitive 
large-scale surveys may help to uncover more distant, low-surface-brightness LG members.

The compilation of LG dwarf star formation histories shows that no two galaxies have the same history, not even within the same morphological type. All LG dwarfs vary widely in star formation histories, metallicity evolution, ages, times and time scales of star formation, and number and distribution of their subpopulations. Many dwarf galaxies show spatial variations in ages and metallicities. Typically star formation lasted longest in the central regions of the galaxies, while in the outer parts often extended old populations are found. Most galaxies appear to share a common epoch of star formation $>10 \mathrm{Gyr}$ ago, but the fractions of these old populations vary and are difficult to age-date. Orbital information would be most valuable in order to search for correlations between star formation episodes and close encounters.

There may be no firm distinction between different morphological types of dwarfs in the LG. Instead the recent detection of young populations in Fornax (Stetson et al. 1998) and Leo I (Gallart et al. 1999) as well as the observation of various transition phases argues in favor of a continuous evolution from low-mass dIrrs to dSphs.

Two modes of star formation are observed in the Local Group: continuous (at constant star formation rate or declining) and episodic star formation. The lack of gas in dEs and dSphs remains puzzling, but possibly associated gas is being detected in the surroundings of some of the dwarfs. According to Lin \& Murray's (1999) scenario gas is present, but photoionized and therefore not detected in $\mathrm{H}$ I studies. Tidal compression at apogalacticon may trigger efficient cooling and onset of star formation, and coincides with the time scales observed in episodic star formation. Observational tests of this scenario are currently being carried out by searching for $\mathrm{H} \alpha$ emission.

Acknowledgements. I thank Puragra Guhathakurta for a fruitful and enjoyable collaboration, Igor Karachentsev, Valentina Karachentseva, and Doug Lin for communications prior to publication, and Taft Armandroff, Claude Carignan, Serge Demers, George Jacoby, and Alan Whiting for valuable discussions. Many thanks to Wolfgang Brandner for his help with IDL. I am indebted to Ian Thompson for a critical reading of the text and for his patience during the process of the writing of this paper. I gratefully acknowledge support by Dennis Zaritsky through NASA LTSA grant NAG-5-3501 and thank him for making it possible for me to attend the Symposium.

This review has made extensive use of NASA's Astrophysics Data System Abstract Service (ADS).

\section{References}

Aparicio, A., García-Pelayo, J.M., Moles, M., Melnick, J. 1987, A\&AS, 71, 297

Aparicio, A., Dalcanton, J.J., Gallart, C., Martínez-Delgado, D. 1997a, AJ, 114, 1447

Aparicio, A., Gallart, C., Bertelli, G. 1997b, AJ, 114, 680

Aparicio, A., Gallart, C., Bertelli, G. 1997c, AJ, 114, 669 
Aparicio, A. 1999, in: Stellar Content of the Local Group, IAU Symp. 192, (eds.)

P. Whitelock \& R. Cannon, ASP Conf. Ser., in press

Armandroff, T.E., Davies, J.E., Jacoby, G.H. 1998, AJ, 116, 2287

Armandroff, T.E., Da Costa, G.S. 1999, in: Stellar Content of the Local Group,

IAU Symp. 192, (eds.) P. Whitelock \& R. Cannon, ASP Conf. Ser., in press

Bellazzini, M., Fusi Pecci, F., Ferraro, F.R. 1996, MNRAS, 278, 947

Bergbusch, P.A., VandenBerg, D.A. 1992, ApJS, 81, 163

Bertelli, G., Bressan, A., Chiosi, C., Fagotto, F., Nasi, E. 1994, A\&A, 106, 275

Bland-Hawthorn, J., Veilleux, S., Cecil, G.N., Putman, M.E., Gibson, B.K., Maloney, P.R. 1998, MNRAS, 299, 611

Blitz, L., Spergel, D.N., Teuben, P.J., Hartmann, D., Burton, W.B. 1999, ApJ, submitted (astro-ph/9803251)

Braun, R., Burton, W.B. 1999, A\&A, accepted (astro-ph/9810433)

Buonanno, R., Corsi, C.E., Zinn, R., Fusi Pecci, F., Hardy, E., Suntzeff, N.B. 1998, ApJ, 501, L33

Burkert, A., Ruiz-Lapuente, P. 1997, ApJ, 480, 297

Byrd, G., Valtonen, M., McCall, M., Innanen, K. 1994, AJ, 107, 2055

Carignan, C. 1985, ApJ, 299, 59

Carignan, C. 1999, in: HI in the Local Universe II, (eds.) R. Webster \& L. Staveley-Smith, PASA, in press

Carignan, C., Beaulieu, S., Côté, S., Demers, S., Mateo, M. 1998, AJ, 116, 1690

Chaboyer, B., Demarque, P., Sarajedini, A. 1996, ApJ, 459, 558

Côté, S., Freeman, K.C., Carignan, C., Quinn, P.J. 1997, AJ, 114, 1313

Da Costa, G.S. 1998, in: Stellar Astrophysics for the Local Group: A First Step to the Universe, (eds.) A. Aparicio \& A. Herrero, CUP, in press

Da Costa, G.S., Armandroff, T.E. 1990, AJ, 100, 162

Da Costa, G.S., Armandroff, T.E., Caldwell, N., Seitzer, P. 1996, AJ, 112, 2576

Demers, S., Irwin, M.J., Kunkel, W.E. 1985, AJ, 90, 1967

De Young, D.S., Heckman, T. 1994, ApJ, 431, 598

Dohm-Palmer, R.C., Skillman, E.D., Saha, A., Tolstoy, E., Mateo, M., Gallagher, J., Hoessel, J., Chiosi, C., Dufour, R.J. 1997, AJ, 114, 2527

Dohm-Palmer, R.C., Skillman, E.D., Gallagher, J., Tolstoy, E., Mateo, M., Dufour, R.J., Saha, A., Hoessel, J., Chiosi, C. 1998, AJ, 116, 1227

Drozdovsky, I.O., Tikhonov, N. 1999, in: Stellar Content of the Local Group, IAU Symp. 192, (eds.) P. Whitelock \& R. Cannon, ASP Conf. Ser., in press

Dunn, A.M., Laflamme, R. 1993, MNRAS, 264, 865

Faber, S.M., Lin, D.N.C. 1983, ApJ, 266, L17

Ferguson, H.C., Binggeli, B. 1994, A\&ARv, 6, 67

Fusi Pecci, F., Bellazzini, M., Cacciari, C., Ferraro, F.R. 1995, AJ, 110, 1664

Gallagher, J.S., Wyse, R.F.G. 1994, PASP, 106, 1225 
Gallagher, J.S., Tolstoy, E., Dohm-Palmer, R.C., Skillman, E.D., Cole, A.A., Hoessel, J.G., Saha, A., Mateo, M. 1998, AJ, 115, 1869

Gallart, C., Aparicio, A., Bertelli, G., Chiosi, C. 1996, AJ, 112, 1950

Gallart, C., Freedman, W.L., Mateo, M., Chiosi, C., Thompson, I.B., Aparicio, A., Bertelli, G., Hodge, P.W., Lee, M.G., Olszewski, E.W., Saha, A., Stetson, P.B., Suntzeff, N.B. 1999, ApJ, 514, in press (astro-ph/9811122) Gardiner, L.T., Noguchi, M. 1996, MNRAS, 278, 191

Geha, M.C., Holtzman, J.A., Mould, J.R., et al. 1998, AJ, 115, 1045

Grebel, E.K. 1997, Reviews in Modern Astronomy, 10, 29

Grebel, E.K., Brander, W. 1998, in: The Magellanic Clouds and Other Dwarf Galaxies, Proc. Bonn/Bochum-Graduiertenkolleg Workshop, (eds.) T.

Richtler \& J.M. Braun, Shaker Verlag, Aachen, p. 151

Grebel, E.K., Guhathakurta, P. 1999, ApJ, 511, in press

Grebel, E.K., Kolatt, Ts., Brandner, W. 1999a, in: Stellar Content of the Local Group, IAU Symp. 192, (eds.) P. Whitelock \& R. Cannon, ASP Conf. Ser., in press

Grebel, E.K., Stetson, P.B. 1999, in: Stellar Content of the Local Group, IAU Symp. 192, (eds.) P. Whitelock \& R. Cannon, ASP Conf. Ser., in press

Grebel, E.K., Zaritsky, D., Harris, J., Thompson, I.B. 1999b, in: New Views of the Magellanic Clouds, (eds.) Y.-H. Chu, N. Suntzeff, J. Hesser, \& D. Bohlender ASP Conf. Ser., in press

Greggio, L., Marconi, G., Tosi, M., Focardi, P. 1993, AJ, 105, 894

Grillmair, C.J., Mould, J.R., Holtzman, J.A., et al. 1998, AJ, 115, 144

Guhathakurta, P., Grebel, E.K., Seitzer, P. 1999, AAS, in press

Han, M., Hoessel, J.G., Gallagher, J.S., Holtzman, J., Stetson, P.S. 1997, AJ, 113,1001

Hodge, P.W. 1989, ARA\&A, 29, 543

Hunter, D.A. 1997, PASP, 109, 937

Hunter, D.A., Plummer, J.D. 1996, ApJ, 462, 732

Hurley-Keller, D., Mateo, M., Nemec, J. 1998, AJ, 115, 1840

Hurley-Keller, D., Mateo, M., Grebel, E.K. 1999, in prep.

Jobin, M., Carignan, C. 1990, AJ, 100, 648

Karachentsev, I.D. 1996, A\&A, 305, 33

Karachentsev, I.D., Makarov, D.A. 1996, AJ, 111, 794

Karachentsev, I.D., Karachentseva, V.E. 1998, in: Dwarf Tales, (eds.) E. Brinks \& E.K. Grebel, Vol. 3, p. 1

Karachentsev, I.D., Makarova, L.N., Andersen, M. 1999, A\&A, submitted

Karachentseva, V.E., Karachentsev, I.D. 1998, A\&AS, 127, 409

Knapp, G.R., Kerr, F.J., Bowers, P.F. 1978, AJ, 83, 360

Koribalski, B., Johnston, S., Otrupcek, R. 1994, MNRAS, 270, L43

Kroupa, P., Bastian, U. 1997, New A, 2, 77

Layden, A.C., Sarajedini, A. 1997, ApJ, 486, L107

Lin, D.N.C., Faber, S.M. 1983, ApJ, 266, L21 
Lin, D.N.C., Murray, S.D. 1999, in: Dwarf Galaxies and Cosmology, XVIII Rencontres des Moriond, (eds.) T.X. Thuân, C. Balkowski, V. Cayatte, \& J.T.T. Vân, Editions Frontières, in press

Lynden-Bell, D., Lynden-Bell, R.M. 1995, MNRAS, 275, 429

MacLow, M.-M., Ferrara, A. 1998, in: The Magellanic Clouds and Other Dwarf Galaxies, Proc. Bonn/Bochum-Graduiertenkolleg Workshop, (eds.) T. Richtler \& J.M. Braun, Shaker Verlag, Aachen, p. 177

Majewski, S.R. 1994, ApJ, 413, 17

Maloney, P.R., Bland-Hawthorn, J. 1999, in: The Galactic Halo, Third Stromlo Symposium, (eds.) B.K. Gibson, T.S. Axelrod \& M.E. Putman, ASP Conf. Ser., in press

Marconi, G., Tosi, M., Greggio, L., Focardi, P. 1995, AJ, 109, 173

Marconi, G., Buonanno, R., Castellani, M., Iannicola, G., Molaro, P., Pasquini, L., Pulone, L. 1998, A\&A, 330, 453

Marconi, G., Buonanno, R., Corsi, C.E., Zinn, R. 1999, in: Stellar Content of the Local Group, IAU Symp. 192, (eds.) P. Whitelock \& R. Cannon, ASP Conf. Ser., in press

Martínez-Delgado, D., Gallart, C., Aparicio, A., Freedman, W. 1999, in: Stellar Content of the Local Group, IAU Symp. 192, (eds.) P. Whitelock \& R. Cannon, ASP Conf. Ser., in press

Massey, P., Armandroff, T.E. 1995, AJ, 109, 2450

Mateo, M. 1998, ARA\&A, 36, 435

Mateo, M., Olszewski, E.W., Volt, S.S., Keane, M.J. 1998, AJ, 116, 2315

Mighell, K.J. 1997, AJ, 114, 1458

Mighell, K.J., Sarajedini, A., French, R.S. 1998, AJ, 116, 2395

Minniti, D., Zijlstra, A.A. 1996, ApJ, 467, L13

Minniti, D., Zijlstra, A.A. 1997, AJ, 114, 147

Minniti, D., Zijlstra, A.A., Victoria Alonso, M. 1999, AJ, in press (astro-ph/9810477)

Montegriffo, P., Bellazzini, M., Ferraro, F.R., Martins, D., Sarajedini, A., Fusi Pecci, F. 1998, MNRAS, 294, 315

Mould, J.R. 1997, PASP, 109, 125

Mould, J.R., Bothun, G.D., Hall, P.J., Staveley-Smith, L., Wright, A.E. 1990, ApJ, 362, L57

Oh, K.S., Lin, D.N.C., Aarseth, S.J. 1995, ApJ, 442, 142

Olsen, K.A.G., Hodge, P.W., Mateo, M., Olszewski, E.W., Schommer, R.A., Suntzeff, N.B., Walker, A.R. 1998, MNRAS, 300, 665

Olszewski, E.W. 1998, in: Galactic Halos: A UC Santa Cruz Workshop, (ed.)

D. Zaritsky, ASP Conf. Ser. 136, p. 70

Oosterloo, T., Da Costa, G.S., Staveley-Smith, L. 1996, AJ, 112, 1969

Philipps, S., Parker, Q., Schwartzenberg, J., Jones, J. 1998, ApJ, 493, L59

Puche, D., Westphal, D. 1994, in: ESO/OHP Workshop on Dwarf Galaxies,

(eds.) G. Meylan \& P. Prugniel, ESO, p. 273

Putman, M.E., Gibson, B.K., Staveley-Smith, L., et al. 1998, Nature, 394, 752 
Richer, M.G., McCall, M.L. 1995, ApJ, 445, 642

Sage, L.J., Welch, G.A., Mitchell, G.F. 1998, ApJ, 507, 726

Sandage, A. 1986, ApJ, 307, 1

Sarajedini, A., Claver, C.F., Ostheimer, J.C. 1997, AJ, 191, 2505

Sarajedini, A., Geisler, D., Harding, P., Schommer, R. 1998, ApJ, 508, L37

Sarajedini, A., Layden, A.C., 1997, AJ, 113, 264

Schlegel, D.J., Finkbeiner, D.P., Davis, M. 1998, ApJ, 500, 525

Schmidt, K.-H., Boller, T. 1992, AN, 313, 189

Scholz, R.-D., Irwin, M.J. 1994, in: Astronomy from Wide-Field Imaging, (eds.)

H.T. MacGillivray et al., Kluwer, p. 535

Schweitzer, A.E., Cudworth, K.M., Majewski, S.R., Suntzeff, N.B. 1995, AJ, 110,2747

Shetrone, M.D., Bolte, M., Stetson, P.B. 1998, AJ, 115, 1888

Skillman, E.D., Bomans, D.J., Kobulnicky, H.A. 1997, ApJ, 474, 205

Smecker-Hane, T., Stetson, P., Hesser, J., Lehnert, M. 1994, AJ, 108, 507

Smith, E.O., Neill, J.D., Mighell, K.J., Rich, R.M. 1996, AJ, 111, 1596

Smith, E.O., Rich, R.M., Neill, J.D. 1998, AJ, 115, 2369

Sofue, Y. 1994, ApJ, 423, 207

Staveley-Smith, L. 1997, PASA, 14, 111

Stetson, P.B., Grebel, E.K. 1999, in: Stellar Content of the Local Group, IAU

Symp. 192, (eds.) P. Whitelock \& R. Cannon, ASP Conf. Ser., in press

Stetson, P.B., Hesser, J.E., Smecker-Hane, T.A. 1998, PASP, 110, 533

Tikhonov, N.A., Karachentsev, I.D. 1999, AstL, submitted

Thuan, T.X., Martin, G.E. 1979, ApJ, 232, L11

Tolstoy, E., Gallagher, J.S., Cole, A.A., Hoessel, J.G., Saha, A., Dohm-Palmer, R.C., Skillman, E.D., Mateo, M., Hurley-Keller, D. 1998, AJ, 116, 1244

Tosi, M., Greggio, L., Marconi, G., Focardi, P. 1991, AJ, 102, 951

Trentham, N. 1998a, MNRAS, 294, 193

Trentham, N. 1998b, MNRAS, 293, 71

Unavane, M., Wyse, R.F.G., Gilmore, G. 1996, MNRAS, 278, 727

van den Bergh, S. 1994, AJ, 107, 1328

van den Bergh, S. 1998, AJ, 116, 1688

van den Bergh, S. 1999, The Local Group of Galaxies, CUP, in prep.

van Dyk, S.D., Puche, D., Wong, T. 1998, AJ, 116, 2341

van Zee, L., Skillman, E.D., Salzer, J.J. 1998, AJ, 116, 1186

Welch, G.A., Sage, L.J., Mitchell, G.F. 1998, ApJ, 499, 209

Wilcots, E.M., Miller, B.W. 1998, AJ, 116, 2363

Whiting, A.B. 1999, AJ, in press

Whiting, A.B., Irwin, M.J., Hau, G.K.T. 1997, AJ, 114, 996

Yahil, A., Tammann, G.A., Sandage, A. 1977, ApJ, 217, 903

Young, L.M., Lo., K.-Y. 1997a, ApJ, 490, 710 
Young, L.M., Lo., K.-Y. 1997b, ApJ, 476, 127

Zaritsky, D., Olszewski, E.W., Schommer, R.A., Peterson, R.C., Aaronson, M. 1989, ApJ, 345, 759

\section{Discussion}

van den Bergh: LGS3 = Pisces and Phoenix might be classified as dSph galaxies that have not yet used up all their gas. How would your (our) conclusion that history correlates with distance from parent galaxy be affected by including Psc and Phx in the dSph sample?

Grebel: The more distant Milky Way dSphs are dominated by intermediateage populations, while the older populations seem to account for only a small fraction of their stellar populations. The generally even more distant potential transition types of $\mathrm{dSph} / \mathrm{dIrr}$ galaxies tend to show prominent old populations, have varying intermediate-age fractions, and some recent star formation. A dominant intermediate-age/young component appears to be lacking in these galaxies, whose presence may be expected from a history/distance relationship. However, other factors such as galaxy mass may play an important role here as well, and there do not seem to be any gas-rich dSph-like galaxies in the vicinity of the large spirals. The dSph/dIrr galaxies may have retained part of their gas due to their larger distance. Their existence does not contradict the ram pressure/tidal stripping history-distance relation that you suggested in 1994.

Hesser: Do these new calculations by Lin et al. help to explain Carignan et al.'s (1998) recent observations of $\mathrm{H}$ I in two distant lobes, in Sculptor?

Grebel: Carignan's detection of the $\mathrm{H}_{\mathrm{I}}$ lobes around Scl presented at the IAU Joint Discussion meeting in August 1997 in Kyoto was one of the triggers for Lin's calculations. The detection seems to fit in well with what Lin et al. suggest since the two lobes outside the optical radius of Scl appear to lie along the general direction of motion of Scl (proper motion from Schweitzer at al. 1996). According to Lin et al. the gas around Scl should remain in the same orbit (even though it may not be bound any more). It may eventually be concentrated in the centre of Scl at apogalacticon and, if it can cool down sufficiently, may form stars if densities get high enough. $\mathrm{H} \alpha$ observations would be very useful to determine if part of the gas may still be photoionized. 